\title{
Emulsion Scanning Technologies
}

\section{Toshiyuki Nakano* and CHORUS collaboration}

Address

Department of Physics, Nagoya University, Japan

E-mail: nnakano@flab.phys.nagoya-u. ac.jpi

\begin{abstract}
Nuclear emulsion is a tracking detector offering three-dimensional information at sub-micron resolutions. To broaden the scope of its application, in particle physics as well as outside, we have been developing an automated readout system. This system, the Ultra Track Selector or UTS, achieves a data taking speed of $1 \mathrm{~cm}^{2} /$ hour. At present, a new system is under development which will further increase the speed by an order of magnitude.
\end{abstract}

\section{Introduction}

The position resolution of nuclear emulsion plates is unsurpassed by any other particle tracking detector. Charged particles traversing the emulsion are recorded as a trail of silver grains, spheres with a diameter of about $0.7 \mu \mathrm{m}$, as indicated in figure the center of grains, an intrinsic resolution of $0.06 \mu \mathrm{m}$ can be achieved since the original size of grains before development is about $0.2 \mu \mathrm{m}$. Figure ${ }_{1}^{\overline{2}}$ shows the distribution of grain residuals with respect to a straight line fit, for the grains produced by a minimum ionizing particle (MIP).

Nuclear emulsion provides truly three-dimensional, rather than projective, information. A MIP gives rise to $30 \sim 40$ grains per $100 \mu \mathrm{m}$, sufficient to measure the track angle for base lengths down to $50 \mu \mathrm{m}$. Angular resolutions of the order of a mrad have been achieved. These features make nuclear emulsion particularly suitable for the detection of short-lived particles such as the tau lepton or charmed hadrons.

\section{Emulsion Readout System}

Tracks in emulsion are measured using a readout system referred to as the Track Selector

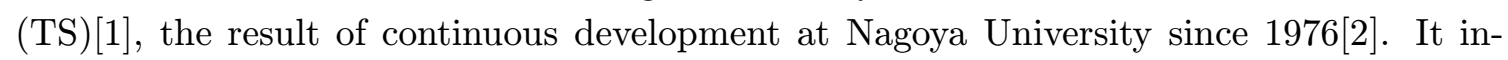

${ }^{*}$ Speaker. 


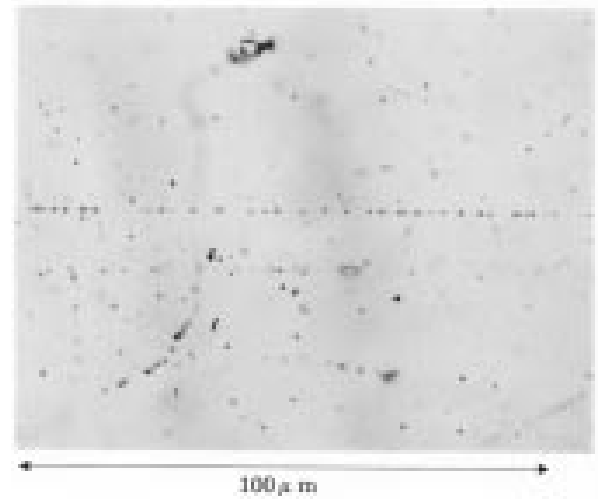

Figure 1: A track of a MIP in nuclear emulsion.

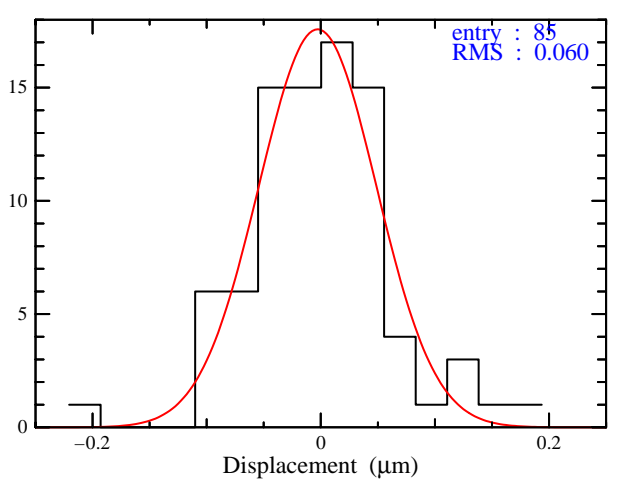

Figure 2: The distribution of residuals for the center of grains.

cludes automated track recognition by dedicated hardware. The CHORUS experiment [3.3ㄱ. performing a $\nu_{\mu} \rightarrow \nu_{\tau}$ oscillation search, is the first large-scale application of this system.

The grain information is collected as tomographic images from a microscope. Using a $50 \times$ objective and a CCD camera capable of a $120 \mathrm{~Hz}$ frame rate, the size of the field of view is $150 \times 120 \mu \mathrm{m}$. Three-dimensional information is obtained by taking 16 images at different focal positions, in the direction perpendicular to the emulsion surface. Since the focal depth is about $3 \mu \mathrm{m}$, a digitized set of images corresponds to a volume of $150 \mu \mathrm{m} \times 120 \mu \mathrm{m} \times 50 \mu \mathrm{m}$.

Figure ${ }_{3}^{\prime} \overline{l_{1}}$ shows a set of images visualized by computer graphics. The volume contains a track which makes a small angle with respect to the vertical axis. The TS shifts successive images such that the track gets to lie along the vertical axis, as indicated in figure Summing up the hit pixels in all transformed images, one obtains the three-dimensional histogram of figure 15 , where the track shows up as a distinct peak. If the track angle is not known a priori, this procedure is repeated for a number of transformations to cover the angular acceptance required. For an angular acceptance of $\pm 400 \mathrm{mrad}$, this may require over 3000 trials.

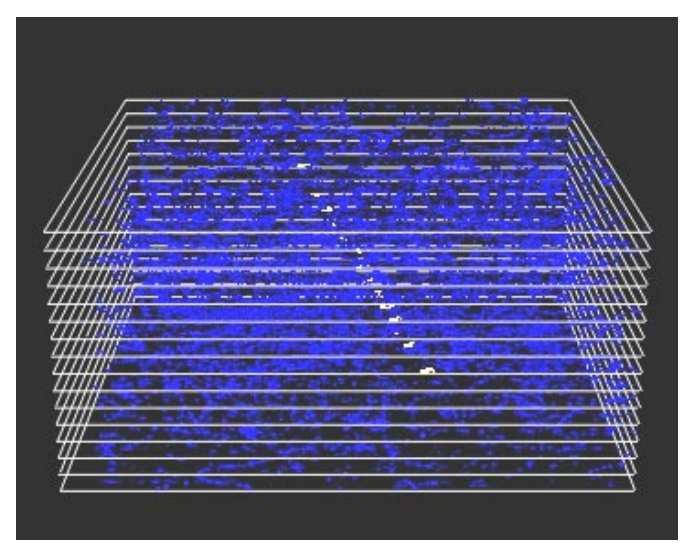

Figure 3: The reconstructed tomographic images of emulsion.

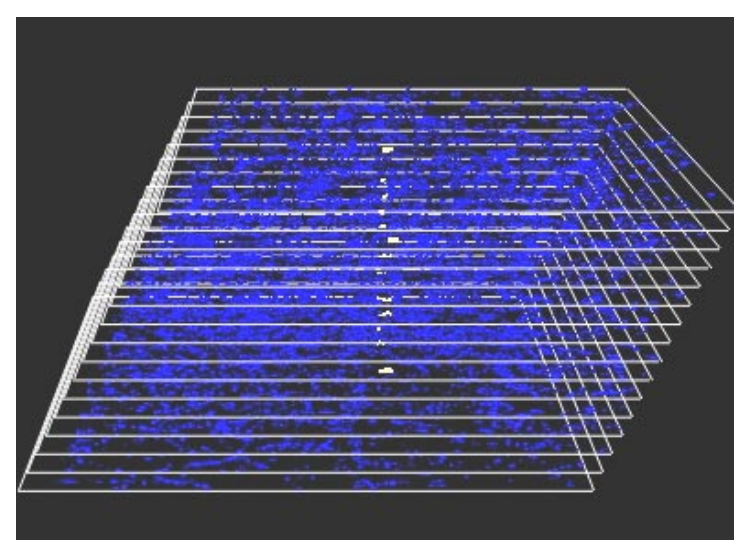

Figure 4: The transformed images. 


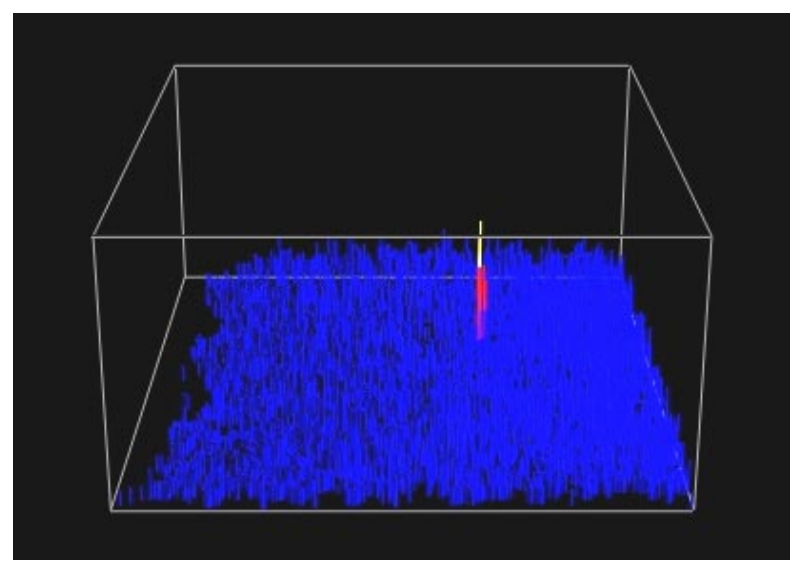

Figure 5: 3D histogram after processing.

\section{Ultra Track Selector}

Real time image processing, especially in three dimensions, requires a considerable amount of computing power. It takes about $0.3 \mathrm{~s}$ to acquire a set of 16 tomographic images, including the time for the stage to move to the next field of view. Ideally, the image processing time should be smaller. An essential prerequisite for fast image processing is sufficient bandwidth. The algorithm of the previous section is performed by the Ultra Track Selector (UTS), a hardware device consisting of up to 50 parallel processing units, each one containing an FPGA and synchronous SRAM. The main clock of the processing unit runs at $120 \mathrm{MHz}$, the memory bandwidth is $480 \mathrm{Mbyte} / \mathrm{s}$. Since the algorithm is hardwired and since there is no dead cycle for memory access, the system achieves a total memory bandwidth of 24 Gbyte/s. The block diagram is shown in figure $\overline{\underline{6}}_{1}^{\prime}$

Each processing unit can search for tracks under a given angle in $\sim 5 \mathrm{~ms}$, corresponding to about 10000 trials per second for a UTS equipped with 50 processing units. Even for angular acceptances up to $\pm 400 \mathrm{mrad}$, the processing time remains comparable to the image taking time. With the application of pipeline processing for image taking and track recognition, the overall throughput of the system can be as large as 3 views/s, corresponding to $\sim 1 \mathrm{~cm}^{2}$ per hour.

The UTS efficiency, shown in figure $\overline{\mathbf{T}}_{1}$, depends slightly on the track angle but remains above $99 \%$ for angles up to $400 \mathrm{mrad}$. The position resolution, illustrated in figure $\overline{8}_{1}$, is characterized by a standard deviation of about $0.2 \mu \mathrm{m}$ in each projection. This is larger than the intrinsic limit for nuclear emulsion because of the error related to the linear scale which defines the stage position.

\section{Scanning System in the future}

At present, the overall throughput is limited by the mechanical response of the stage. Improving this would allow the use of a faster camera but is difficult because of inertia. For example, the time to move from one view to the next is about $100 \mathrm{~ms}$, largely determined 


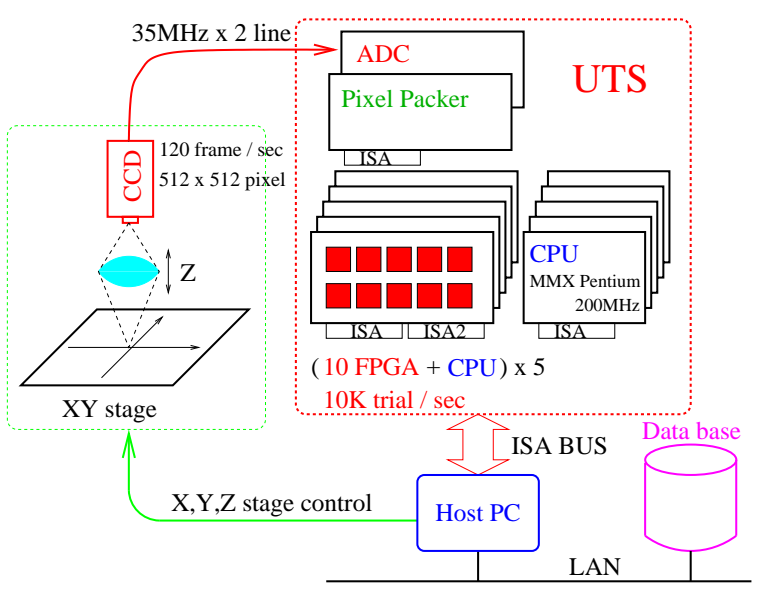

Figure 6: The UTS block diagram.

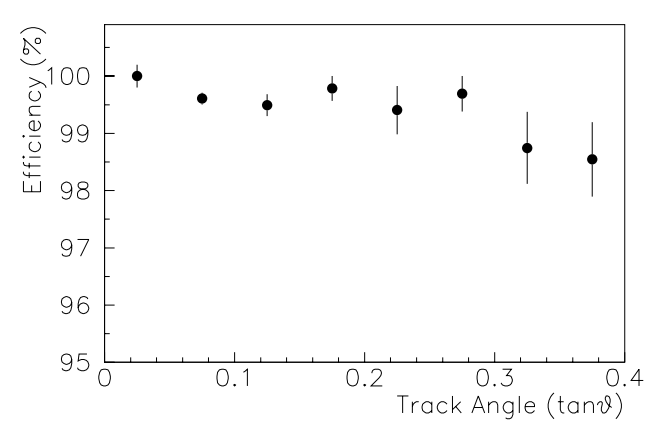

Figure 7: Track finding efficiency.
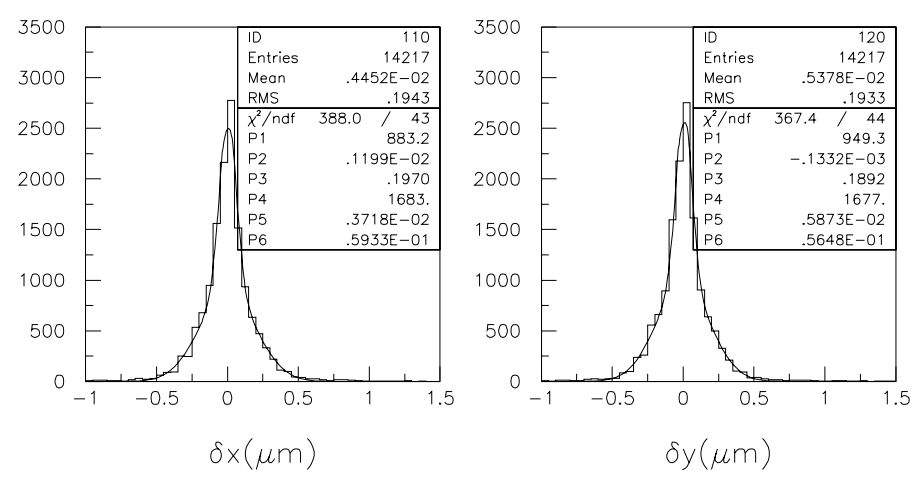

Figure 8: Position resolution for the UTS track measurement.

by the time to reach a complete stop. To overcome this problem, we are developing a new optical unit based on an actuator to move the objective. This will allow the stage to move continuously rather than to stop between sets of tomographic images, avoiding the step motion altogether. In the transverse plane, the objective lens moves in the same direction at the same velocity as the stage. This procedure, illustrated in figure $\underline{\underline{9}}$, is similar 
to a follow-shot in photography: the synchronization of the objective lens and the stage is equivalent to having the stage at rest when the image is taken. The method has been successfully tested for the prototype of a new objective with piezo electric actuator. The new system now under development, called the Super-UTS (S-UTS), will employ a high speed CCD camera capable of 3000 frames per second to achieve an overall throughput of $20 \mathrm{~cm}^{2}$ per hour.

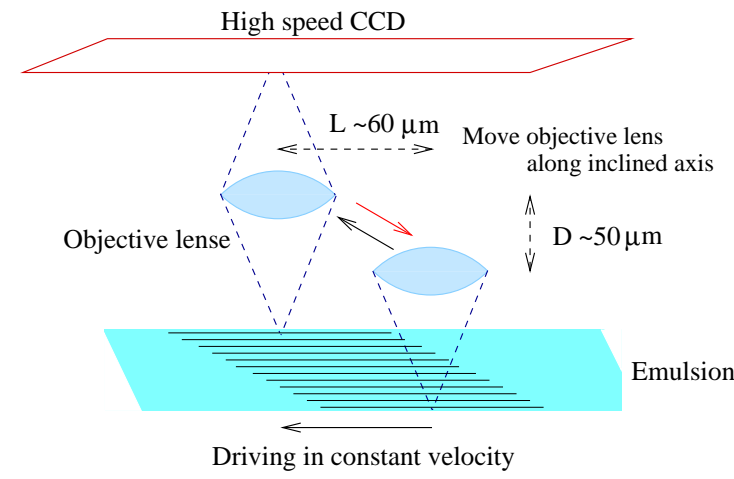

Figure 9: The follow-shot method for emulsion readout.

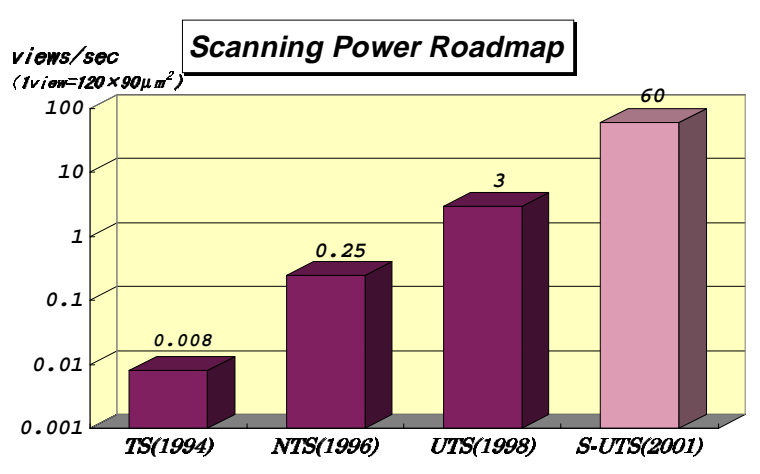

Figure 10: The increase in the readout speed over the past couple of years.

\section{Conclusions}

As shown in figure $1 \overline{0} 0_{0}^{\prime \prime}$ over the past decade the speed of nuclear emulsion readout has increased by an order of magnitude every two years. This has made the Net Scan Method possible, a technique relying on the recognition of all tracks in a volume of several $\mathrm{mm}^{3}$ up to a few $\mathrm{cm}^{3}$ around the interaction point to obtain a complete reconstruction of the event topology. Using this method, the CHORUS oscillation search experiment has been able to extend its scope to the study of charm physics. The technique also allowed the discovery of tau neutrino interactions, by the DONUT experiment in 1998 [i] in parallel, the S-UTS development is well under way. The S-UTS system will be essential to the success of the OPERA experiment [i $[\bar{b}]$, a long baseline oscillation search at Gran Sasso using the CNGS neutrino beam.

\section{References}

[1] S. Aoki et al., №ucl. Instrum. Meth. B $51-19 \overline{9} 0)^{-} \overline{4} 6 \overline{6}$

[2] K. Hoshino et al, Proc. of International Cosmic ray Sympo. of High Energy Phenomena., Tokyo 1974

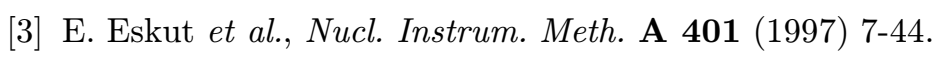

[4] K. Kodama et al., 'Phys. Lett. B.504 20012 21 $18-224$.

[5] M. Guler et al., CERN SPSC 2000-028, July 2000. 